\title{
A SURVEY ON TRAFFIC SIGN RECOGNITION AND DETECTION
}

\author{
Albert Keerimolel, Sharifa Galsulkar, Brandon Gowray* \\ Final Year UG Students, Department of Computer Engineering, Xavier Institute of Engineering \\ Mahim Causeway, Opp. Raheja Hospital, Mumbai 400016, India. \\ *Corresponding author brandongowray@gmail.com
}

\begin{abstract}
Classifying traffic signs is an indispensable task for autonomous driving systems. Depending on the country, traffic signs possess a wide variability in their visual appearance making it harder for classification systems to succeed. Either the classifier should be fine-tuned or a bigger collection of images should be used. Nowadays, Intelligent Autonomous Vehicles together with Advanced Driver Assistance Systems (ADAS) deal with the problem of traffic sign recognition. It is a challenging real world computer vision problem due to the different and complex scenarios they are placed into. After images are classified they are made into the groups like highway signs, speed signs, danger signs etc.
\end{abstract}

Keywords - Self-Driving Car, traffic sign, recognition and detection, HOG and SVM, MSER and HSV.

\section{INTRODUCTION}

Traffic sign detection and recognition (TSDR) system has been introduced to reduce traffic safety concerns. A TSDR system detects and recognizes traffic signs from and within images captured by cameras or imaging sensors displaying to the user the user what traffic rules are applicable at that stretch of road. In bad traffic conditions, the driver may not notice traffic signs, due to which accidents occur. In such cases, the TSDR system comes into play. The main objective of the research on TSDR is to improve the efficiency and working speed of the TSDR system. To develop a TSDR system is a difficult job given the continuous changes in the environment and lighting conditions. There are other issues such as partial obscuring, multiple traffic signs appearing at the same time, and blurring and fading of old traffic signs, which can also create problem for the detection purpose and need to be addressed. For application of TSDR system in real-time environment such as selfdriving cars, a quick algorithm is required. Also dealing with issues, a recognition system should avoid errors in recognition of non-signs.

\section{Problem Definition}

Classifying traffic signs is a very important task for autonomous driving systems as the safety of everyone as well as the passenger depends on it. Depending on the country, traffic signs possess a variety in their visual appearance making it harder for classification systems to succeed.

Nowadays, Intelligent Autonomous Vehicles together with Advanced Driver Assistance Systems
(ADAS) deal with the problem of traffic sign recognition. It is a challenging real world computer

vision problem due to the different and complex scenarios they are placed into. The proposed system will help understand the problem and provide a systematic way of approaching the problem.

\section{AIMS \& OBJECTIVES}

All Through this project we aim to make a system which can be used universally for traffic signs and symbols, which in turn would help us learn more about deep learning. The objectives of the system is first to detect and then classify signs. To detect, a combination of MSER and HSV is used. This works well when we have lots of images as well as not clear ones. For classification HOG is used, along with SVM Classifier. Now this working in conjunction make the results more accurate and we get a model with high accuracy.

\section{IV.SCOPE}

The traffic signs focus on reducing traffic and increasing safety of the road network through various travel management measures, speed limits etc. Traffic signs should remove the encroachments, congestion and improve the traffic signal, road condition and geometrics features at intersections. The traffic signs should be as a guidance or speaker on a road network. Traffic sign reduce the traffic congestion along the road and also provide facilities for the road users. 
Road signs tell users of the various regulations when using the road as well as providing guidance and when needed also warnings for safe, efficient operation. The purpose of road signs is to promote road safety and effective use of roads by both pedestrians and traffic.

\section{Literature SURVEY}

The first research on traffic sign recognition was back in 1987; Akatsuka and Imai tried to make a very basic traffic sign recognition system. A system capable of by itself recognize of traffic sign and used as an assistance for drivers, telling them about the presence of some specific restriction or danger in speeding or construction work. It can be used to provide the automatic recognition for specific traffic signs. Generally, the procedure of a traffic sign recognition system is divided into two parts, detection and classification.

\section{A. Detection}

The aim in using traffic sign detection is to locate the regions of interest (ROI) in which a traffic sign is likely to be found. Cropping the excess space is what's required to get the main sign. This technique called as ROI. ROI locates the traffic sign in based on its shape dimensions etc. The traffic signs are cropped which is useful. The background image is removed as it is not an area of interest. By this we assume, that a large part of the image area can be ignored as not required. Traffic signs are designed with set color and shape making them easier to differentiate and recognize.

\section{B. Classification}

In this part the sign is classified based on the type shape colour and the information the sign is giving.
Histogram of oriented gradients (HOG) Features: HOG decomposes an image into small squared cells, computes a histogram of oriented gradients in each cell, normalizes the result using a block-wise pattern, and return a descriptor for each cell
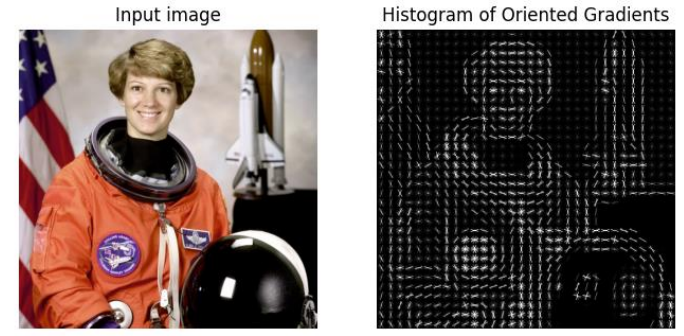

Fig. 1 A sample of HOG

We can also use UNet for classification with an encoder decoder architecture. In this the image is given through various layers and then the output is also put through layers before final output is given.

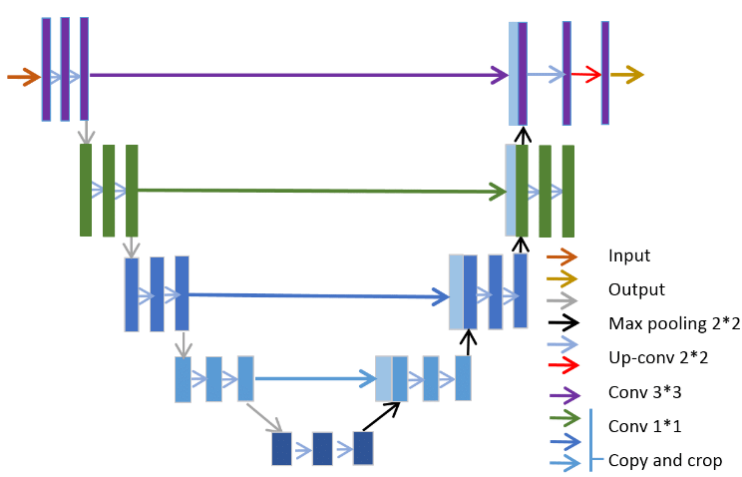

Fig. 2 UNet Architecture 


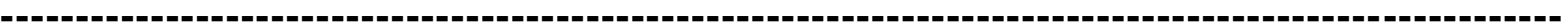 \\ and valuable suggestion it would not have had the idea to develop the project.}

\section{Proposed System}

Through this project we aim to make a system which can be used universally for traffic signs and symbols, which in turn would help us learn more about deep learning. The objectives of the system is first to detect and then classify signs. To detect, localization U-Net, a convolutional neural network with an encoder-decoder architecture, is used. We can also use image preprocessing techniques such as median filter to clean salt and pepper noise in image, then contrast normalization over each channel. For classification SqueezNet can be used but for greater accuracy we can also use HOG classifier. Since this is a survey paper we are still looking out for the better method from the above proposed systems to get the best accuracy and result.

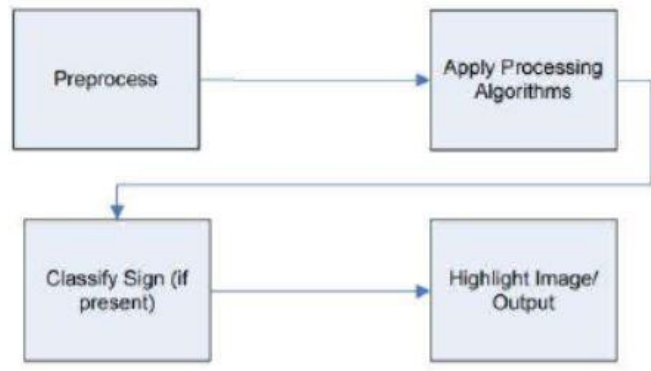

Fig. 3 Block Diagram

\section{CONCLUSION}

From doing this project we plan to understand the science behind auto recognition of traffic signs helping autonomous cars and drivers to be safer when driving on the roads. We also get to learn the various aspects of image processing and computer vision used algorithms to get desired output. We plan to future study this topic and prepare a full working traffic sign detection and recognition system.

\section{ACKNOWLEDGMENT}

Authors wish to acknowledge "Mr. Vijay Jumb" our guide who gave us his valuable suggestions and ideas when we were in need of them. He encouraged us to work on this project. We are immensely grateful to all involved in this project as without their inspiration

\section{REFERENCES}

[1] "Recognition of Traffic Signs" by Sayed Omar Sadat, Vishal Kumar Pal, Kshitij Jassal, International Journal of Scientific research and Review, Volume 07, Issue 03, March 2019.

\section{[2] https://en.wikipedia.org/wiki/Traffic- Sign recognition}

[3] "An Automatic Traffic Sign Detection and Recognition System Based on Colour Segmentation, Shape Matching, and SVM" by Safat b. Wali, Mahammad A. Hannan, Aini Hussain, and Salina A. Samad, Hindawi Journal.

\section{[4] German Traffic Sign Database from Keggle}

[5]. "Study on traffic sign recognition" by Mohamed Yussof Radzak, International Journal of Research Studies in Computer Science and Engineering. 